\title{
The 2018 Aged Care National Antimicrobial Prescribing Survey: results show room for improvement
}

\section{Leslie Dowson ${ }^{1,2}$ Arjun Rajkhowa ${ }^{2,3}$ Kirsty Buising ${ }^{2,3,4}$ David CM Kong ${ }^{1,2,5}$ Rhonda L Stuart ${ }^{1,2,6}$ Karin Thursky2,3,7 Noleen Bennett ${ }^{2,3,8}$ \\ 'Monash University ${ }^{2}$ National Centre for Antimicrobial Stewardship, Melbourne \\ ${ }^{3}$ University of Melbourne \\ ${ }^{4}$ Victorian Infectious Diseases Service, Melbourne \\ ${ }^{5}$ Ballarat Health Services, Ballarat \\ ${ }^{6}$ Monash Health, Clayton ${ }^{7}$ Peter MacCallum Cancer Centre, Melbourne \\ ${ }^{8}$ Victorian Healthcare Associated Infection Surveillance System Coordinating Centre, Melbourne \\ Victoria}

Full author details are available online.

\section{Keywords}

aged, antibiotic prophylaxis, antibiotics, antimicrobial stewardship, homes for the aged, infection

Aust Prescr 2019;42:200-203 https://doi.org/10.18773/ austprescr.2019.066

First published

14 November 2019

\section{SUMMARY}

The annual Aged Care National Antimicrobial Prescribing Survey aims to identify local and national prescribing issues and guide antimicrobial stewardship goals.

In the 2018 point prevalence survey, medication charts of over 20,000 residents were reviewed from 407 participating facilities across Australia.

On the day of the survey, almost $10 \%$ of residents were prescribed an antimicrobial.

Nearly two-thirds of recently prescribed antimicrobials were for residents who had no documented signs or symptoms of infection.

Over a quarter of antimicrobials had been prescribed for longer than six months.

Incomplete documentation was a prominent barrier to proper review of antimicrobial therapy, with the indication, review date or stop date not documented for many prescriptions.

Recommendations include using appropriate microbiological testing to guide prescribing, following national antimicrobial prescribing guidelines, documenting the indication for the antimicrobial, and its start, stop and review dates, and monitoring and re-evaluating long-term antimicrobial use.

\section{Introduction}

Residents of aged-care homes are especially vulnerable to infection.' Lack of diagnostic certainty can compound the complexity of decision making around antimicrobial use. ${ }^{2}$ In aged-care homes, antimicrobial use takes place within a context of heightened risk and vigilance due to the potential for complications and clinical deterioration.

Overuse and inappropriate use of antimicrobials, however, can accelerate antimicrobial resistance and increase morbidity and mortality from drug-resistant infections. Inappropriate use of antimicrobials can directly and indirectly harm residents, ${ }^{3}$ and agedcare homes can serve as reservoirs and sites of transmission of drug-resistant organisms. ${ }^{4,5}$ Agedcare homes play an important role in communityhospital transmission of drug-resistant organisms, ${ }^{6}$ so prudent use of antimicrobials is a necessity. Surveillance of antimicrobial use can guide improved prescribing practices.

\section{The 2018 Aged Care National Antimicrobial Prescribing Survey}

The Aged Care National Antimicrobial Prescribing Survey (AC NAPS) is an annual survey of infections and antimicrobial prescribing practices in Australian aged-care homes and multipurpose services. ${ }^{7}$ Its primary aim is to assist participating facilities to reduce infections and improve their use of antimicrobials.

AC NAPS is a point prevalence survey. De-identified data are collected from residents' medical records and entered into an online AC NAPS database by participating nurses, pharmacists or infection-control nurse consultants. The first survey was piloted nationally in $2015,{ }^{8}$ and was followed by surveys in $2016,{ }^{9} 2017^{10}$ and $2018^{11}$. The survey has consistently highlighted aspects of antimicrobial use in aged-care facilities that could be improved.

In 2018, the medication charts of 20,030 permanent, respite or transitional aged-care residents from 407 facilities were reviewed. Facilities from every state and territory in Australia participated. Victorian, state-government-operated, and major city and innerregional facilities contributed proportionally more data to the survey than their actual representation in the Australian aged-care sector.

\section{Significant results of the survey}

On the day of the survey, the following results were found:

- $\quad$ Almost $10 \%(1988 / 20,030)$ of residents were prescribed at least one antimicrobial while only $2.9 \%$ $(581 / 20,030)$ had signs or symptoms of infection. 
- Nearly 65\% (64.6\%, 1009/1563) of recently prescribed antimicrobials were for residents who did not have documented signs or symptoms of suspected infection in the week before they started treatment.

- Over a quarter $(28.3 \%, 663 / 2341)$ of antimicrobials had been administered for longer than six months.

- Topical antimicrobials made up over one-third (36.3\%, 849/2341) of antimicrobials prescribed.

- Incomplete documentation was a prominent barrier to proper review of medicines. The indication for the antimicrobial was not documented in a quarter of prescriptions (25.1\%, 587/2341), and the review date or stop date was not documented for $58.9 \%$ (1380/2341) of prescriptions.

- $\quad$ Other - skin, soft tissue or mucosal (18.3\%, 428/2341), cystitis (16\%, 375/2341) and pneumonia $(9.4 \%, 221 / 2341)$ were the three most common indications presumed or documented for antimicrobials prescribed.

- Cefalexin (20.3\%, 475/2341) was the most commonly prescribed antimicrobial, followed by clotrimazole (19\%, 444/2341).

These findings show there is scope for improvement. The discrepancy between the proportion of residents who were prescribed antimicrobials and the proportion who were identified as having signs or symptoms of infection presents a potential target for quality improvement. In the latter group, the proportion of residents whose suspected infections met infection criteria was only $22.1 \%$ (346/1563).

The observed widespread practice of prolonged antimicrobial use (including for prophylaxis) was surprising and suggests that more frequent review and re-evaluation of antimicrobial therapy is required. The findings of the survey may relate to wider system issues such as fragmented access to visiting medical staff and lack of continuity of care. ${ }^{12}$

The findings also point to priority infections in this setting and the antimicrobials that are being used for treatment. The results could be used to help guide clinical education on these specific conditions.

\section{How does practice compare to guidelines?}

Empiric antimicrobial use should be in accordance with recommendations in endorsed national prescribing guidelines. ${ }^{13,14}$ However, non-concordant use frequently occurs in many Australian aged-care homes. For example, for community-acquired lower respiratory tract infections, amoxicillin with clavulanic acid is repeatedly chosen as a first-line antimicrobial by some prescribers, ${ }^{15}$ despite it being recommended as second-line therapy. For skin and soft tissue infections, antimicrobial choices are generally concordant with endorsed national prescribing guidelines when an indication is documented. However, most often, prescribers do not document the indication. ${ }^{16}$

\section{Recommendations to improve antimicrobial use}

General principles for improving antimicrobial use in aged-care homes include the following:

- All health professionals should have easy access to endorsed national prescribing guidelines. ${ }^{13,14}$ Therapeutic Guidelines: Antibiotic has recently been updated. ${ }^{13}$ These should be used to guide antimicrobial prescribing.

- Advance care planning documentation should be consulted, as necessary. ${ }^{13}$

- Clinical care in aged-care homes should meet the Antimicrobial Stewardship Clinical Care Standard. ${ }^{7}$

- The indication for antimicrobial use, and start, stop and review dates should all be clearly documented in the resident's medical record. ${ }^{17}$

- Antimicrobial review plans and actions, including monitoring the resident's clinical condition, reviewing the results of any investigations and appropriately adjusting any therapy, should be documented in the resident's medical record and followed. ${ }^{17}$

- Prolonged antimicrobial use should be avoided. If it is required, residents should be closely monitored and their therapy regularly re-evaluated. ${ }^{17}$

- System-wide issues regarding access to, and continuity of, medical care for aged-care home residents should be addressed. ${ }^{12}$

While local issues can be identified through participation in the AC NAPS, there are also some well-researched and widely known issues with antimicrobial use in aged-care homes that have been identified more broadly. One such issue is the frequent and unnecessary testing of urine specimens, which can lead to unnecessary antimicrobial prescribing. 18,19 In aged-care homes, urinalysis and urine cultures are only appropriate when a resident has symptoms of a urinary tract infection. This may include specific and non-specific symptoms. ${ }^{13}$ Cloudy or malodorous urine alone is not a sufficient reason to perform urinalysis or urine cultures, or to prescribe an antimicrobial. ${ }^{13}$ 
Recommendations to improve antimicrobial use for common infections in aged care are listed in the Table. Links to essential resources for antimicrobial stewardship initiatives have been compiled by the Australian Government. ${ }^{20}$

\section{Standards for antimicrobial stewardship}

With the newly updated Aged Care Quality Standards, Australian aged-care homes are now required to demonstrate that they have infectioncontrol practices in place, and 'practices to promote appropriate antimicrobial prescribing and use to support optimal care. ${ }^{21}$ It is hoped that more aged-care homes will incorporate antimicrobial stewardship into their quality and safety framework, and actively engage in surveillance and other quality improvement activities.

Quality agencies have also been promoting the implementation of antimicrobial stewardship programs in aged-care homes through the Antimicrobial Stewardship Clinical Care Standard. ${ }^{17}$ This provides guidance on the quality of care that residents and families should expect to receive for an infection. It includes recommendations about antimicrobial use and treatment, such as the use of broad-spectrum antibiotics, and the review of treatment.

\section{Conclusion}

Improving the safety and quality of care in the agedcare sector is a national priority. ${ }^{22}$ It is important that the quality use of medicines is consistently promoted through existing and emerging quality improvement paradigms.

By participating in the AC NAPS survey, each facility can generate customised reports and examine their local issues. These reports may serve as a basis for educating staff, residents and their families about antimicrobial use and provide an incentive to make clinical policy and practice changes. They can be presented to accreditation organisations as evidence of quality improvement initiatives. Considered together, these approaches are anticipated to yield better outcomes for residents. $<$

\section{David Kong has sat on advisory boards for Becton Dickinson and MSD, and has received financial travel support from MSD, all of which was unrelated to the current work.}

The Aged Care National Antimicrobial Prescribing Survey is coordinated by the National Centre for Antimicrobial Stewardship in partnership with the Guidance Group (Melbourne Health) and the VICNISS Coordinating Centre (Melbourne Health). It is supported by the Australian Commission on Safety and Quality in Health Care as part of the Antimicrobial Use and Resistance in Australia project. ${ }^{7}$

\section{Table Recommendations to improve antimicrobial use for common infections in aged-care homes}

\begin{tabular}{ll} 
Infection & Recommendation \\
\hline Urinary tract & Urinalysis and urine cultures are only appropriate when a resident has symptoms of a urinary tract infection, such as \\
acute dysuria. ${ }^{13}$ & Cloudy or malodorous urine alone is not a sufficient reason to perform urinalysis or urine cultures, or to prescribe \\
an antimicrobial..$^{13}$ & Comprehensive investigation and treatment algorithms for urinary tract infections in aged-care homes are available. ${ }^{13}$ \\
- Cefalexin is no longer the antimicrobial of choice for acute cystitis. ${ }^{13}$ The recommended first-line empiric therapy in \\
non-pregnant women and men is trimethoprim or nitrofurantoin. ${ }^{13}$ If cefalexin is prescribed, the dosing and frequency of \\
administration should be concordant with the recommendations. ${ }^{13,14}$
\end{tabular}

Respiratory tract

- Comprehensive guidance on managing respiratory tract infections in aged-care home residents is available.13,14

- Polymerase chain reaction testing in residents with signs and symptoms of influenza-like illness or lower respiratory tract infection is recommended. ${ }^{13,15}$

- Amoxicillin with clavulanic acid should be reserved as a second-line antimicrobial for community-acquired lower respiratory tract infection. ${ }^{13}$ The recommended first-line empiric therapy for community-acquired lower respiratory tract infection is amoxicillin (or doxycycline or cefuroxime for residents with penicillin hypersensitivity). ${ }^{13}$

Skin, soft tissue and mucosal
- The indication for any antimicrobial use, including creams and drops, should be properly documented. 16,17

- If topical antimicrobials are initiated 'when required' by the nursing staff, residents should be reviewed by a medical doctor. ${ }^{17}$ 


\section{REFERENCES}

1. Australian Institute of Health and Welfare. Older Australia at a glance. Canberra: AlHW; 2018. https://www.aihw.gov.au/ reports/older-people/older-australia-at-a-glance/contents/ health-aged-care-service-use [cited 2019 Nov 1]

2. Nicolle LE, Yoshikawa TT. Urinary tract infection in longterm-care facility residents. Clin Infect Dis 2000;31:757-61. https://doi.org/10.1086/313996

3. Daneman N, Bronskill SE, Gruneir A, Newman AM, Fischer HD, Rochon PA, et al. Variability in antibiotic use across nursing homes and the risk of antibiotic related adverse outcomes for individual residents. JAMA Intern Med 2015;175:1331-9. https://doi.org/10.1001/ jamainternmed.2015.2770

4. Lim CJ, Cheng AC, Kennon J, Spelman D, Hale D, Melican G, et al. Prevalence of multidrug-resistant organisms and risk factors for carriage in long-term care facilities: a nested casecontrol study. J Antimicrob Chemother 2014:69:1972-80. https://doi.org/10.1093/jac/dku077

5. Stuart RL, Kotsanas D, Webb B, Vandergraaf S, Gillespie EE, Hogg GG, et al. Prevalence of antimicrobial-resistant organisms in residential aged care facilities. Med J Aust 2011;195:530-3. https://doi.org/10.5694/mja11.10724

6. Wang J, Foxman B, Mody L, Snitkin ES. Network of microbial and antibiotic interactions drive colonization and infection with multidrug-resistant organisms. Proc Natl Acad Sci USA 2017;114:10467-72. https://doi.org/10.1073/pnas.1710235114

7. Australian Commission on Safety and Quality in Health Care. Antimicrobial prescribing in Australian residential aged-care. Sydney: ACSQHC; 2019. https://www.safetyandquality.gov.au/ our-work/antimicrobial-resistance/antimicrobial-use-andresistance-australia-surveillance-system-aura/antimicrobialprescribing-australian-residential-aged-care [cited 2019 Nov 1]

8. National Centre for Antimicrobial Stewardship and Australian Commission on Safety and Quality in Health Care. Antimicrobial prescribing and infections in Australian residential aged care facilities: results of the 2015 Aged Care National Antimicrobial Prescribing Survey pilot. Sydney: Commonwealth of Australia; 2016

https://www.safetyandquality.gov.au/publications-andresources/resource-library/antimicrobial-prescribing-andinfections-australian-residential-aged-care-facilities-results2015-aged-care-national-antimicrobial-prescribing-surveypilot [cited 2019 Nov 1]

9. National Centre for Antimicrobial Stewardship and Australian Commission on Safety and Quality in Health Care. Aged Care National Antimicrobial Prescribing Survey report 2016. Sydney: ACSQHC; 2017. https://www.safetyandquality.gov.au/ publications-and-resources/resource-library/2016-agedcare-national-antimicrobial-prescribing-survey-report [cited 2019 Nov 1]

10. National Centre for Antimicrobial Stewardship and Australian Commission on Safety and Quality in Health Care. Antimicrobial prescribing and infections in Australian aged care homes: results of the 2017 Aged Care National Antimicrobial Prescribing Survey. Sydney: ACSQHC; 2018. https://www.safetyandquality.gov.au/publications-andresources/resource-library/2017-aged-care-nationalantimicrobial-prescribing-survey-report [cited 2019 Nov 1]
11. National Centre for Antimicrobial Stewardship and Australian Commission on Safety and Quality in Health Care. Antimicrobial prescribing and infections in Australian aged care homes: results of the 2018 Aged Care National Antimicrobial Prescribing Survey. Sydney: ACSQHC; 2019. https://www.safetyandquality.gov.au/publications-andresources/resource-library/2018-aged-care-nationalantimicrobial-prescribing-survey-report [cited 2019 Nov 1]

12. Lim CJ, Kwong M, Stuart RL, Buising KL, Friedman ND, Bennett $N$, et al. Antimicrobial stewardship in residential aged care facilities: need and readiness assessment. BMC Infect Dis 2014;14:410. https://doi.org/10.1186/1471-2334-14-410

13. Antibiotic. In eTG complete [Internet]. Melbourne: Therapeutic Guidelines Ltd; 2019. www.tg.org.au [cited 2019 Nov 1]

14. AMH Aged Care Companion [Internet]. Adelaide: Australian Medicines Handbook Pty Ltd; 2019. https://agedcare.amh.net.au [cited 2019 Nov 1]

15. Dowson L, Marshall C, Buising K, Friedman ND, Kong DC, Stuart RL. Optimizing treatment of respiratory tract infections in nursing homes: nurse-initiated polymerase chain reaction testing. Am J Infect Control 2019;47:911-5. https://doi.org/10.1016/j.ajic.2019.02.001

16. Bennett NJ, Imam N, Ingram RJ, James RS, Buising KL, Bull AL, et al. Skin and soft tissue infections and current antimicrobial prescribing practices in Australian aged care residents. Epidemiol Infect 2019;147:e87. https://doi.org/ $10.1017 /$ S0950268819000128

17. Australian Commission on Safety and Quality in Health Care. Antimicrobial stewardship clinical care standard. Sydney: ACSQHC; 2014. https://www.safetyandquality.gov.au/ our-work/clinical-care-standards/antimicrobial-stewardshipclinical-care-standard [cited 2019 Nov 1]

18. Abrutyn E, Mossey J, Berlin JA, Boscia J, Levison M, Pitsakis P. et al. Does asymptomatic bacteriuria predict mortality and does antimicrobial treatment reduce mortality in elderly ambulatory women? Ann Intern Med 1994;120:827-33. https://doi.org/10.7326/0003-4819-120-10-199405150-00003

19. Nicolle LE, Mayhew WJ, Bryan L. Prospective randomized comparison of therapy and no therapy for asymptomatic bacteriuria in institutionalized elderly women. Am J Med 1987;83:27-33. https://doi.org/10.1016/0002-9343(87)90493-1

20. Antimicrobial stewardship [Internet]. Canberra: Antimicrobial Resistance, Australian Government; 2017. https://www.amr.gov.au/what-you-can-do/aged-care/ antibiotic-stewardship [cited 2019 Nov 1]

21. Aged care quality standards. Canberra: Australian Government Department of Health; 2019 https://agedcare.health.gov.au/quality/aged-care-qualitystandards [cited 2019 Nov 1]

22. Aged care reforms. Canberra: Australian Government Department of Human Services; 2019. https://www.humanservices.gov.au/organisations/healthprofessionals/subjects/aged-care-reforms [cited 2019 Nov 1]

\section{FURTHER READING}

NPS MedicineWise Learning. Urinary tract infections in residential aged-care facilities. Sydney: NPS MedicineWise; 2019. https://learn.nps.org.au/mod/page/view.php?id=6026 [cited 2019 Nov 1] 